\title{
DIAGNÓSTICO DEL DÉFICIT DE CAPACIDAD INSTITUCIONAL DE LOS PROCESOS PARA EL REDISEÑO ORGANIZACIONAL EN EL SECTOR PÚBLICO
}

\author{
DIAGNOSIS OF INSTITUTIONAL CAPACITY DEFICIT FOR \\ PUBLIC SECTOR ORGANIZATIONAL REDESIGN
}

Víctor Beltrán Saravia'

\section{RESUMEN}

El Sistema de Análisis de Capacidad Institucional $(\mathrm{SADCl})$ es una metodología desarrollada por el Dr. Alain Tobelem cuando se desempeñaba como consultor del Banco Mundial (BIRF). El SADCI ha sido aplicado en numerosos países y en diferentes tipos de proyectos, particularmente en América Latina. En la mayoría de los casos, la metodología se utilizó en proyectos con financiamiento del Banco Interamericano de Desarrollo (BID) y del Banco Mundial (BIRF).

Sin embargo, el SADCI puede también ser aplicado para conocer el déficit de capacidad en la gestión rutinaria de una organización, así como para evaluar los resultados de un programa o proyecto.

Los principales aportes de esta metodología son la identificación de los déficits de capacidad institucional (en adelante, $\mathrm{DCl}$ ), su clasificación en diferentes tipos de déficits atendiendo a sus posibles causas, y la formulación de un componente de desarrollo institucional (en adelante, CDI) como una respuesta de fortalecimiento e intervención organizacional para la superación de esos déficits.

Se aplicará esta metodología para evaluar el déficit de capacidad institucional de un Gobierno Regional del Perú en relación a un Modelo de Gestión por Resultados. Esta evaluación se realizará sobre la base de la determinación de la criticidad de los procesos de gestión del MGXR y del déficit de capacidad del Gobierno Regional para gestionarlos.

Palabras clave: Sistema de Análisis de Capacidad Institucional (SADCI), déficit de capacidad institucional (DCI), componente de desarrollo institucional (CDI)

\begin{abstract}
SADCI (Analysis System Institutional Capacity) is a conceptual development by Dr. Alain Tobelem, while serving as a consultant to the World Bank (IBRD). It has been applied in many countries and in different types of projects, particularly in Latin America. In most cases, the methodology used in projects financed by the Interamerican Development Bank (IDB) and World Bank (IBRD). However, the SADCI can also be applied to determine the capacity deficit in the routine management of an organization, and to evaluate the results of a program or project. The main product of the methodology is to identify the lack of institutional capacity (hereinafter DCI), classification into different types of deficits in line with their possible causes, and formulating an institutional development component (hereinafter $\mathrm{CDI}$ ) as a response to strengthening and organizational intervention to overcome these deficits. This methodology was used to assess the lack of institutional capacity of one Peruvian Regional Governments, in relation to a Results Management Model, based on the determination of the criticality of processes MGXR management and capacity deficits Regional government to manage them.
\end{abstract}

Keywords: Analysis System Institutional Capacity $(S A D C I)$, lack of institutional capacity (DCI), institutional development component (CDI)

\footnotetext{
1 Ingeniero Industrial, Magíster en Ingeniería Industrial, Consultor, Director de la Escuela de Ingeniería Industrial de la Facultad de Ingeniería, Universidad Ricardo Palma, Av. Benavides 5440, Lima, Perú. <vbeltrans@hotmail.com>
} 


\section{INTRODUCCIÓN}

La metodología SADCI se aplica en los casos en que se necesita identificar el grado de capacidad institucional actual para llevar a cabo determinadas funciones, evaluar los obstáculos y debilidades a remover o eliminar, y establecer las acciones y planes requeridos para ello. En tal sentido, su dimensión temporal de referencia es el futuro y su aplicación está concebida particularmente para programas y proyectos. La situación típica es el análisis del componente de fortalecimiento institucional requerido para asegurar el éxito de un proyecto. Así fue concebido inicialmente este instrumento. Además, aun cuando el propósito original de esta metodología no lo contemplaba expresamente, el enfoque SADCI es también compatible con el análisis de situaciones en las que el presente y el pasado pasan a ser dimensiones temporales relevantes y, a la vez, con casos en los que el objeto de estudio no es ya un programa o proyecto, sino la gestión ordinaria de una institución.

\section{TIPOS DE DÉFICITS DE CAPACIDAD INSTITUCIONAL}

Se trata de identificar diferentes obstáculos que podrían impedir la conclusión de la tarea. Representan un déficit en la medida en que existe una brecha perceptible entre lo que la tarea implica y lo que se afirma que se hará (lo que se desea hacer). Esa brecha de capacidad de la institución o actor es la que debe identificarse y ponderarse.

Es útil señalar que las acciones y esfuerzos encarados por los responsables de las organizaciones para cerrar esa brecha es una condición sine qua non: si no existen esfuerzos en pro del fortalecimiento en diferentes aspectos por parte de las organizaciones, no puede alcanzarse el objetivo general.

Naturalmente, esas brechas de capacidad no responden exclusivamente a factores intraorganizacionales, sino que pueden deberse a restricciones contextuales o estructurales que la institución no está en condiciones de cambiar. Existen diversos factores que provocan problemas organizacionales, tales como los déficits relacionados con leyes, normas y "reglas de juego", con la estructura organizacional interna y distribución de funciones, con la capacidad financiera y física de las agencias ejecutoras,

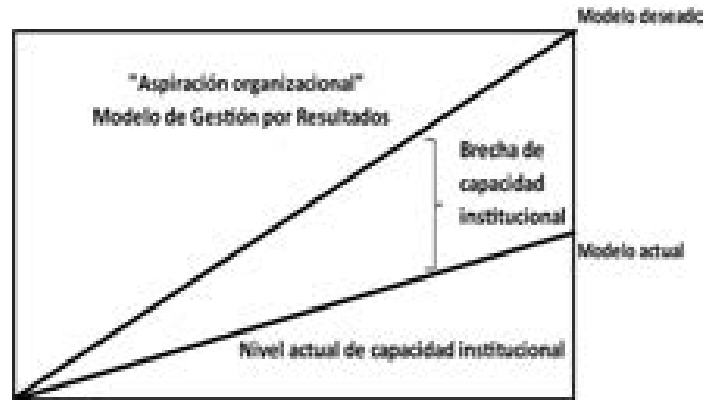

Gráfico 1: Diagrama de brechas por el déficit de capacidad con políticas de personal y sistema de recompensas, con la capacidad individual de los participantes en las agencias involucradas en el proyecto, con relaciones interinstitucionales, entre otros.

En el siguiente gráfico, se muestra el enfoque de brechas que se originan por los déficits de capacidad institucional.

\section{DEL MODELO ORGANIZACIONAL ACTUAL}

El marco legal que norma sobre los Gobiernos Regionales lo otorga la Ley N 27867. Los Gobiernos Regionales están constituidos por el Consejo Regional (órgano normativo y fiscalizador), que incluye al Presidente Regional, el Vicepresidente Regional y los Consejeros Regionales de las provincias de cada región. Estos representantes son elegidos mediante voto directo por cuatro años. 
La Presidencia Regional cuenta con una Secretaría Regional y cinco Gerencias Regionales coordinadas por una Gerencia Regional.

La Administración Regional se ejerce bajo un sistema gerencial y se sustenta en la planificación estratégica, organización, dirección, ejecución, evaluación y control dentro del marco de las normas emitidas por los sistemas administrativos nacionales.

Contrariamente a los criterios organizativos óptimos, las organizaciones de la Administración Pública, entre ellos los Gobiernos Regionales, se encuentran estructurados según el principio de departamentalización funcional, el cual considera que las funciones establecidas en la norma de creación de una dependencia u organismo deben representarse biunívocamente en la estructura organizativa. Incluso, en algunos casos, la misma norma pretende definir la estructura, por lo que deja de lado los aspectos estratégicos propios de la región (visión, misión, valores, estrategias, objetivos estratégicos, iniciativas estratégicas, etc.). Se trataría, pues, de una estructura orgánico-funcional tradicional que no se sustenta en procesos.

Debe entenderse que la estructura orgánica es un instrumento para lograr los fines de la entidad y no un fin en sí mismo. Por lo tanto, su diseño y reingeniería deben relacionarse con las estrategias (donde están incluidos los resultados), los procesos y la asignación de recursos. Por este motivo, resulta imprescindible que la entidad pública posea las capacidades teóricas, metodológicas y técnicas que le permitan definir, instrumentar y actualizar sus planes estratégicos de corto, mediano y largo plazo.

Se considera que un diseño organizacional vinculado a resultados $y$ orientados a la atención del cliente debe tener la siguiente secuencia lógica.

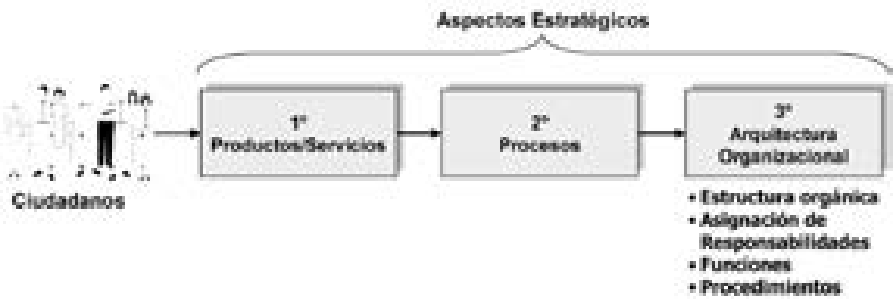

Gráfico 2: Secuencia del diseño organizacional

Primero, se deben tener definidos los productos y servicios que brinda el organismo. A partir de ello, se realiza el despliegue de los procesos (producción institucional) necesarios para alcanzarlos cumpliendo con estándares de calidad. Finalmente, se elabora la arquitectura organizacional adecuada para gestionar los procesos y el organismo de manera eficaz y eficiente. Todo esto debe estar alineado a los aspectos estratégicos de manera que se asegure la obtención de los resultados planteados y comprometidos.

El diagnóstico organizacional del Gobierno Regional busca identificar los procesos característicos del modelo organizacional deseado (Modelo de

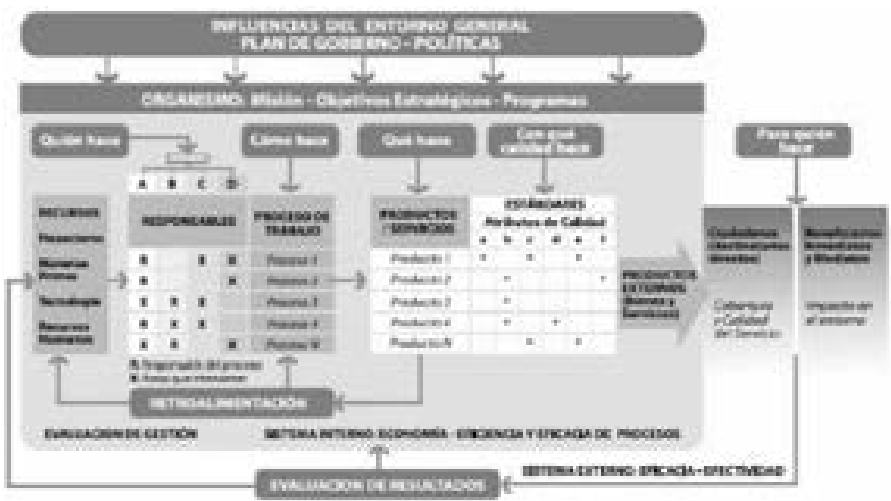

Gráfico 3: Servicios, productos y procesos Gestión por Resultados) y determinar las brechas con el modelo organizacional actual. 


\section{DEL MODELO DE GESTIÓN POR RESULTADOS: ASPIRACIÓN ORGANIZACIONAL}

La gestión por resultados es un modelo efectivo de planificación y control que tiende a dotar a la Administración Pública de un conjunto de metodologías y técnicas diseñadas para lograr la consistencia y coherencia buscadas entre los objetivos estratégicos y los planes de cada uno de los organismos.

Al considerar el desempeño organizacional en función de los resultados y de la atención al ciudadano en lugar de hacerlo en referencia al cumplimiento de normas y procedimientos, este enfoque implica un cambio sustancial en las modalidades de gestión que tradicionalmente se aplican en las Administraciones Públicas. Orientar la gestión a resultados implica supeditar las

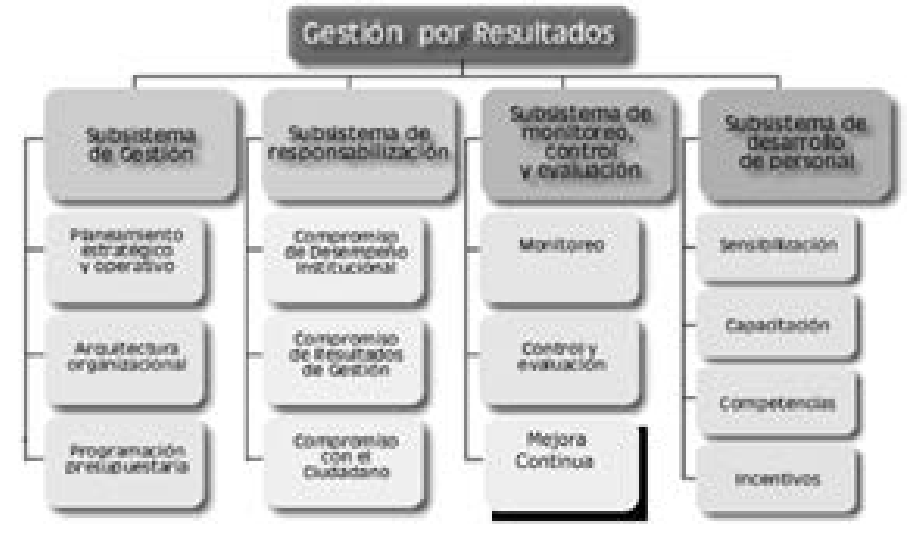

Gráfico 4: Modelo de Gestión por Resultados estructuras, los procesos, los procedimientos, los controles (dentro de ellos, la propia ley) y la administración de los recursos con los que cuenta un organismo a resultados establecidos y comprometidos previamente entre las instancias de decisión y control intervinientes.

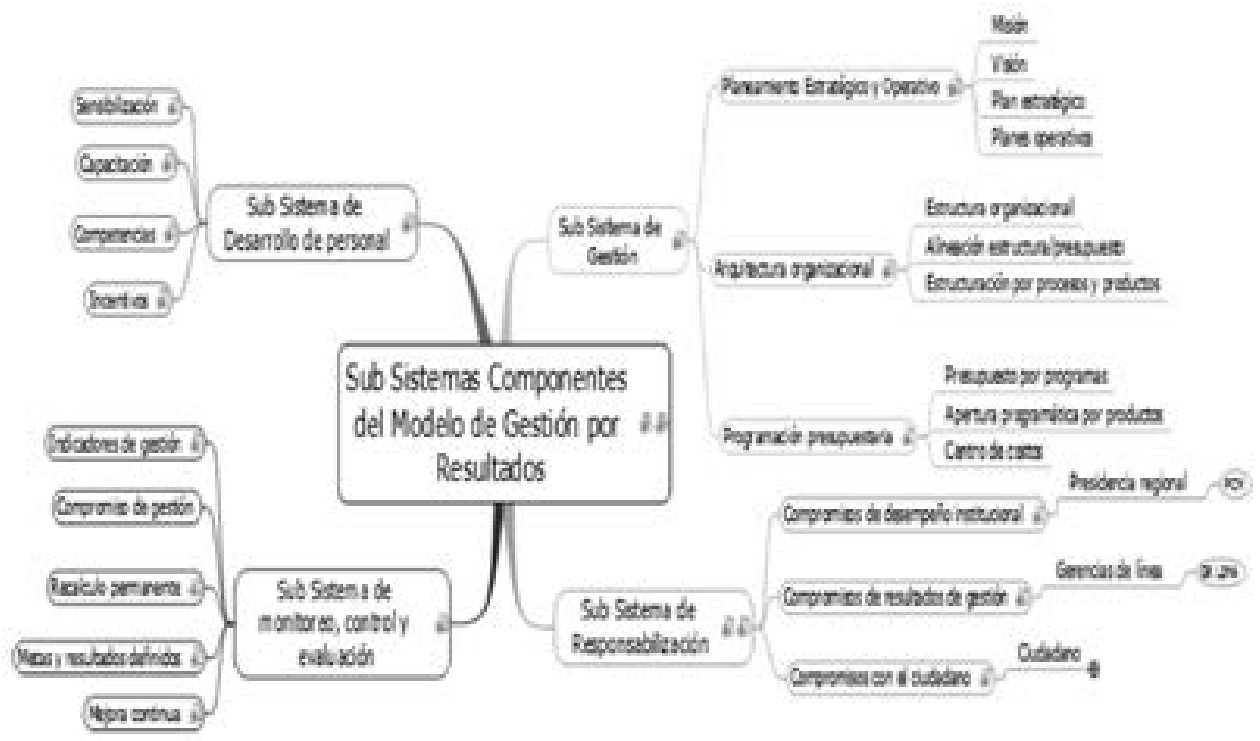

\section{Sistemas de Informadión}

Gráfico 5: Subsistemas del Modelo de Gestión por Resultados 
La gestión por resultados implica, además, crear un modelo capaz de establecer cómo se alcanzarán los resultados propuestos, puesto que no es suficiente indicar qué se quiere lograr, sino también cómo se va a lograr. En ese sentido, se considera que la gestión por procesos, la calidad total, la cultura organizacional y la implementación de sofisticados sistemas de información se ubican como complementos naturales para lograr un Modelo de Gestión por Resultados eficaz y eficiente. El modelo propuesto se forma de cuatro subsistemas, tal como se aprecia en el esquema siguiente.

Para una mayor comprensión del alcance y cobertura del Modelo de Gestión por Resultados, se muestra un gráfico en el que se agrupa a sus componentes.

A continuación, se muestra y describe los siguientes ejes rectores: transparencia, calidad de servicios, optimización de recursos (productividad), participación y control ciudadano, e innovación tecnológica. Todos estos encuentran soporte en la direccionalidad estratégica.

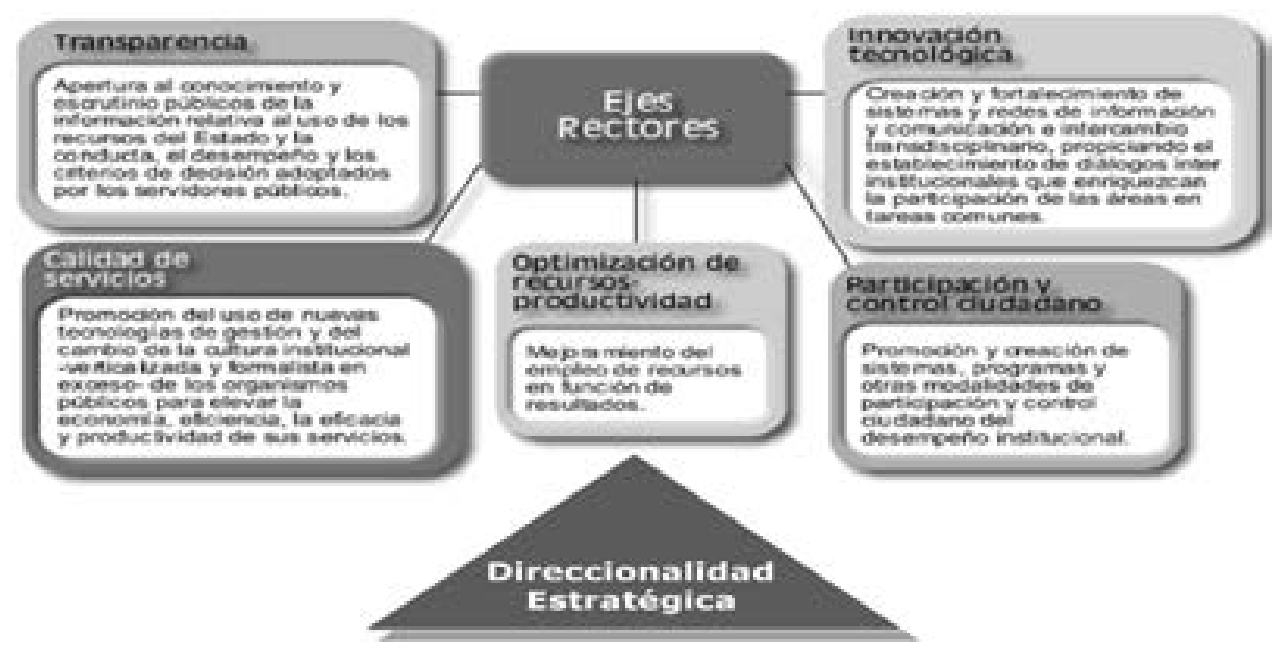

Gráfico 6: Ejes rectores del Modelo de Gestión por Resultados

\section{EL TABLERO DE CONTROL DEL DÉFICIT DE CAPACIDAD INSTITUCIONAL (DCI)}

El tablero de control DCI permite analizar el estado de la capacidad institucional en base a la criticidad de los procesos y la capacidad para llevarlos a cabo. El análisis de los motivos del déficit, a su vez, permite identificar las causas humanas y organizacionales y sus vinculaciones. El tablero DCI permite analizar la visión de los usuarios sobre el estado del déficit de capacidad institucional según procesos de gestión de diferentes ámbitos, tales como unidades o áreas de instituciones, redes institucionales, programas, proyectos o partes de los anteriores. La capacidad institucional es el potencial de las organizaciones para producir valor para terceros o desarrollarse ellas mismas. El déficit (DCI) se expresa en las siguientes dos dimensiones.

- Criticidad de los procesos es la estimación del perjuicio potencial para el ámbito institucional analizado que se ocasionaría por el deficiente logro de los resultados requeridos de los procesos.

- Déficit de capacidad es la estimación de la falta de capacidad institucional en el ámbito analizado para lograr, adecuada y oportunamente, los resultados de los procesos tomando en cuenta tanto los factores internos e institucionales como las restricciones contextuales existentes. 
TABLA 1: DEFINICIÓN DE PROCESOS EN EL SADCI

\begin{tabular}{|c|c|c|c|}
\hline PROCESOS BAJO CONTROL & \multicolumn{2}{|c|}{ PROCESOS EN TRANSICIÓN } & $\begin{array}{l}\text { PROCESOS CON ALTO DÉFICIT DE } \\
\text { CAPACIDAD INSTITUCIONAL }\end{array}$ \\
\hline $\begin{array}{l}\text { Bajo riesgo institucional: } \\
\text { procesos poco críticos y bajo } \\
\text { control }\end{array}$ & $\begin{array}{l}\text { Alerta por criticidad de } \\
\text { procesos: procesos críticos y } \\
\text { bajo control }\end{array}$ & $\begin{array}{l}\text { Alerta por baja capacidad: } \\
\text { procesos poco críticos y fuera } \\
\text { de control }\end{array}$ & $\begin{array}{c}\text { Déficit crítico de capacidad } \\
\text { institucional: procesos críticos y } \\
\text { fuera de control }\end{array}$ \\
\hline $\begin{array}{l}\text { Los procesos de gestión son } \\
\text { de baja criticidad, por lo que, } \\
\text { en caso de mal desempeño en } \\
\text { estos, los perjuicios potenciales } \\
\text { son bajos. Al mismo tiempo, la } \\
\text { unidad cuenta con la capacidad } \\
\text { necesaria y suficiente para el } \\
\text { logro de los resultados. }\end{array}$ & $\begin{array}{l}\text { Los procesos de gestión son de } \\
\text { alta criticidad y, por lo tanto, } \\
\text { en caso de mal desempeño en } \\
\text { estos, los perjuicios potenciales } \\
\text { son muy altos, pero la unidad } \\
\text { cuenta con capacidad necesaria } \\
\text { y suficiente para el logro de los } \\
\text { resultados correspondientes. }\end{array}$ & $\begin{array}{l}\text { La unidad no cuenta con capa- } \\
\text { cidad para llevar a cabo ade- } \\
\text { cuadamente los procesos de } \\
\text { gestión. Pero estos son de baja } \\
\text { criticidad y, por lo tanto, los } \\
\text { perjuicios que se ocasionarían } \\
\text { por no lograr de los resulta- } \\
\text { dos esperados no afectan de } \\
\text { manera significativa el normal } \\
\text { funcionamiento organizacional } \\
\text { del ámbito analizado. }\end{array}$ & $\begin{array}{l}\text { La unidad no cuenta con la ca- } \\
\text { pacidad necesaria y suficiente } \\
\text { para el logro de los resultados } \\
\text { en los procesos de gestión } \\
\text { más críticos, cuyo déficit oca- } \\
\text { siona los mayores perjuicios, } \\
\text { tanto en lo que hace a los pro- } \\
\text { pios procesos como en otros } \\
\text { ámbitos relacionados. }\end{array}$ \\
\hline
\end{tabular}

\section{IDENTIFICACIÓN DE DÉFICITS DE CAPACIDADES INSTITUCIONALES}

Se proyectó una visión organizacional del Gobierno Regional de Lima para alcanzar un nuevo Modelo de Gestión por Resultados.

Se efectuó la descomposición del Modelo de Gestión por Resultados en sus procesos, los cuales se toman como críticos. Bajo este criterio, si el Gobierno Regional no aspira a mejorar sus metas bajo una nueva forma de gestión por resultados, que involucra eficiencia, indicadores, metas, mejoramiento continuo sobre la base de una cultura de calidad, administración basada en procesos, se estaría ocasionando un perjuicio potencial para sus propias aspiraciones de logros orientados hacia el servicio al ciudadano.

Ante esa visión prospectiva que significa un Modelo de Gestión por Resultados, se confrontan las capacidades actuales de la gestión y procesos del Gobierno Regional para determinar el déficit de capacidad tomando en cuenta tanto los factores internos e institucionales como las restricciones contextuales existentes.

Aplicando el método DELPHI con la opinión de los consultores especialistas, se estableció la siguiente escala:
$\triangle$ de 0 a 4 para los procesos bajo control;
de 4 a 6 para los procesos en transición;
$\ominus$ de 6 a 10 para los procesos con alto déficit de capacidad.

Luego, se procedió a la valoración considerando el estado actual de gestión del GR y la criticidad de los procesos del Modelo de Gestión por Resultados. Con la valoración, se determina un ranking de los procesos más deficitarios hacia los procesos menos deficitarios.

Con la valoración de los procesos, se pasa a mapear, en el tablero, aquellos procesos de bajo control, los de zona intermedia y los críticos. 


\begin{tabular}{|c|c|c|c|c|c|c|c|}
\hline \multicolumn{2}{|c|}{ PROCESOS DE MODELO DE GESTIÓN POR RESULTADOS } & \multicolumn{5}{|c|}{ VALORACIÓN } & \multirow{3}{*}{$\begin{array}{l}\frac{0}{0} \\
0 \\
0 \\
0 \\
* \\
* \\
0\end{array}$} \\
\hline \multirow{2}{*}{ Código } & \multirow{2}{*}{ Descripción } & \multirow{2}{*}{ 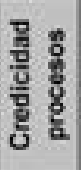 } & \multirow{2}{*}{$\begin{array}{l}\frac{\mathrm{g}}{\mathrm{c}} \\
\frac{\mathrm{c}}{\mathrm{c}} \\
\frac{\mathrm{c}}{\mathrm{c}} \\
\frac{\mathrm{c}}{\mathrm{s}}\end{array}$} & \multirow{2}{*}{ 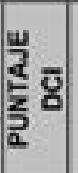 } & \multirow{2}{*}{ 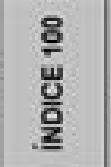 } & \multirow{2}{*}{ हू } & \\
\hline & & & & & & & \\
\hline SDP2 & Capacitación & 10 & 10 & 10 & 156.25 & 1 & \\
\hline SDP4 & Incentivos & 10 & 9 & 9.5 & 148.44 & 2 & \\
\hline SDP1 & Sensibilización & 9 & 8 & 8.5 & 132.81 & 3 & \\
\hline SDP3 & Competencias & 9 & 7.5 & 8.3 & 128.91 & 4 & \\
\hline SDM3 & Mejora continua & 8 & 8 & 8 & 125.00 & 5 & \\
\hline SDM2 & Control y evaluación & 9 & 6 & 7.5 & 117.19 & 6 & \\
\hline SDR2 & Compromiso de resultados de gestión & 6 & 6 & 6 & 93.75 & 7 & \\
\hline SDM1 & Monitoreo & 6 & 5 & 5.5 & 85.94 & 8 & \\
\hline SDG2 & Arquitectura organizacional & 6 & 4 & 5 & 78.13 & 9 & \\
\hline SOR1 & Compromiso de desempeno institucional & 5 & 5 & 5 & 78.13 & 10 & \\
\hline SDR3 & Compromiso con el cludadano & 5 & 4 & 4.5 & 70.31 & 11 & \\
\hline SDG1 & Planeamiento estrategico operativo & 3 & 3 & 3 & 46.88 & 12 & \\
\hline SDG3 & Programación presupuestaria & 3 & 2 & 2.5 & 39.06 & 13 & \\
\hline
\end{tabular}

Gráfico 7: Valoración de procesos SADCI

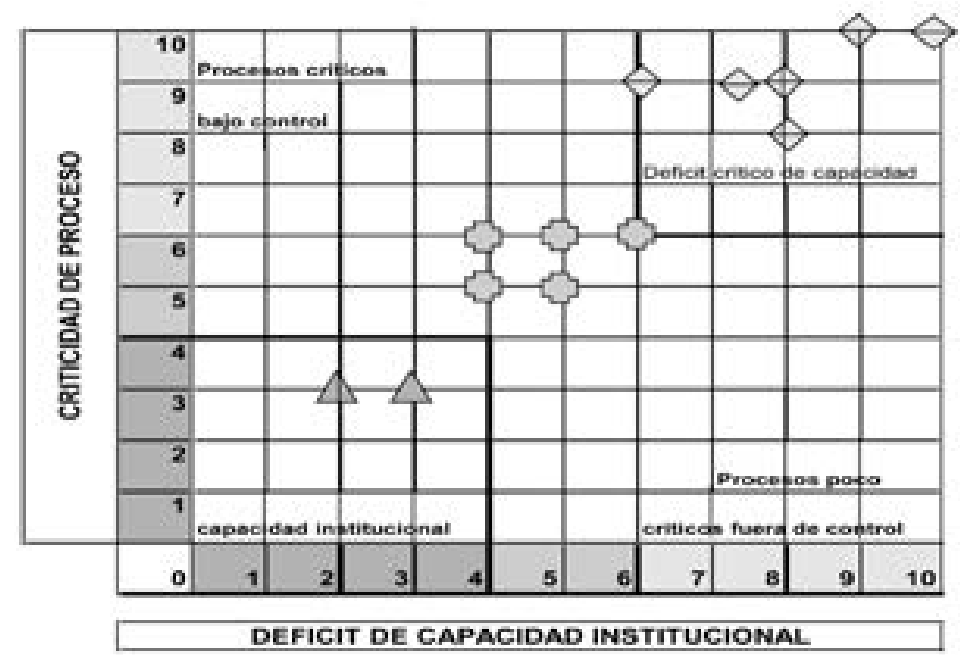

Gráfico 8: Mapeo de procesos SADCI

\section{CONCLUSIONES Y RECOMENDACIONES}

La identificación de los procesos críticos (en zona roja) nos permite observar que estos requieren de una mayor atención en el desarrollo de un nuevo modelo organizacional de gestión por resultados del Gobierno Regional.

En tal sentido, si se desea orientar el cambio hacia un Modelo de Gestión por Resultados (escala 10), el proceso de capacitación es altamente crítico en el modelo organizacional actual.

Del diagnóstico realizado con el especialista en sistemas administrativos, se comprobó que el GR no disponía de presupuesto para capacitación ni de un plan de capacitación. En consecuencia, el proceso de capacitación actual, en el GR, es altamente deficitario e ingresa a la zona de déficit crítico de 
capacidad institucional, por lo que se establece un gap o brecha entre el proceso de capacitación actual y el proceso de capacitación deseado.

La identificación de los procesos bajo control (en zona verde) permite conocer las fortalezas en la planificación estratégica y en la formulación y control presupuestario. Esto es posible, particularmente, por la introducción del Presupuesto por Resultados, el cual plantea el diseńo de acciones en función a la resolución de problemas críticos que afectan a la población y requiere de la participación de diversos actores que alinean su accionar en tal sentido.

Los procesos bajo incertidumbre (en zona ámbar) permiten identificar que se debe hacer un esfuerzo por lograr su control. Para esto, el proceso de gestión por resultados se hace clave y su tránsito es muy cercano a los procesos críticos ubicados en la zona roja.

En el alineamiento de los ejes estratégicos al Modelo de Gestión por Resultados, se observa que los procesos del subsistema de monitoreo, control y evaluación no tienen la importancia necesaria en el Plan de Desarrollo Concertado 2007-2021, por lo que se concluye que se trata de un proceso crítico nuevo.

Según el concepto de alineamiento organizacional, desarrollado por David Norton y Robert Kalan, las claves para alinear a todas y cada una de las partes de una organización o institución deben estar bajo un mismo "paraguas estratégico".

Para reducir las brechas o gaps entre el modelo organizacional actual y el modelo organizacional deseado, se elabora un plan progresivo de cambios.

Para los procesos deficitarios, se debe establecer un programa de acción para su mejora. En este caso, se propone un plan mínimo de capacitación y asistencia técnica orientado hacia el cambio organizacional para alcanzar el Modelo de Gestión por Resultados.

La falta de una cultura de procesos y de calidad provoca ineficiencias en la gestión del Gobierno Regional.

Es necesario revisar el establecimiento de los contratos de gestión por resultados al interior del Gobierno Regional sobre la base de los indicadores y metas internas que se definen en el Modelo de Gestión por Resultados. Esta experiencia se aplica en el ámbito de las empresas productivas del Estado. Es conveniente transparentar las responsabilidades dentro del Gobierno Regional. 